\title{
Subcellular localization of KL-6 mucin in colorectal carcinoma cell lines: Association with metastatic potential and cell morphology
}

\author{
QIAN GUO $^{1,2}$, WEI TANG ${ }^{1}$, YOSHINORI INAGAKI ${ }^{1,3}$, NORIHIRO KOKUDO ${ }^{1}$, YASUHIKO SUGAWARA ${ }^{1}$, \\ HIRONA KARAKO ${ }^{1}$, MUNEHIRO NAKATA $^{3}$ and MASATOSHI MAKUUCHI ${ }^{1}$ \\ ${ }^{1}$ Hepato-Biliary-Pancreatic Surgery Division, Department of Surgery, Graduate School of Medicine, \\ the University of Tokyo, Tokyo, Japan; ${ }^{2}$ Basic Research Center of Oncology, Shandong Tumor Hospital, \\ Shandong, Jinan, P.R. China; ${ }^{3}$ Department of Applied Biochemistry, Tokai University, Kanagawa, Japan
}

Received November 17, 2006; Accepted December 27, 2006

\begin{abstract}
KL-6 mucin, a type of MUC1 mucin, is expressed in many malignant tissues including colorectal adenocarcinoma. Previous studies on colorectal adenocarcinoma tissues showed that subcellular localization of KL-6 mucin was associated with the tumor metastatic potential and the patient prognosis. In the present study, to further investigate the significance of subcellular localization of KL-6 mucin, we examined KL-6 mucin expression in colorectal carcinoma cell lines, COLO 201, LoVo, WiDr, and DLD-1, by means of Western blot, flow cytometric and immunocytochemical analyses in conjunction with xylitol treatment. COLO 201 cells characterized by free cells in culture and high metastatic potential revealed extremely high level expression of KL-6 mucin in the cytoplasm and cell surface. The cell surface mucin of COLO 201 cells could not be removed by xylitol treatment, suggesting that the mucin may tightly bind to the cell membrane. LoVo cells characterized by adhesive cells in culture and high metastatic potential express a low level of KL-6 mucin in their cytoplasm and cell surface. In contrast, WiDr and DLD-1 cells, both of which are characterized by low metastatic potential, express KL-6 mucin around the cell surface. The mucin of WiDr and DLD-1 cells could be removed by xylitol treatment, suggesting that KL-6 mucin of these two cell lines may be weakly attached around the cell surface. These results suggest that expression level and subcellular localization of KL-6 mucin may be related to the cell morphology in culture and metastatic potential in colorectal carcinoma cell lines. In addition, xylitol is an
\end{abstract}

Correspondence to: Dr Wei Tang, Hepato-Biliary-Pancreatic Surgery Division, Department of Surgery, Graduate School of Medicine, the University of Tokyo, 7-3-1 Hongo, Bunkyo-ku, Tokyo 113-8655, Japan

E-mail: tang-sur@h.u-tokyo.ac.jp

Key words: KL-6 mucin, subcellular localization, colorectal carcinoma cell line, metastasis, cell morphology effective tool to remove KL-6 mucin weakly attached around the cell surface and to investigate the role of subcellular localization of KL-6 mucin.

\section{Introduction}

MUC1 mucin is abundantly expressed at the surface of epithelial cells in many tissues (1-4). MUC1 expression is also observed in carcinomas that arise in several parts of the body such as the colon, breast, lung, pancreas, papillary thyroid, and gallbladder (5-8). The deduced amino acid sequence of MUC1 mucin reveals four distinct domains: the $\mathrm{NH}_{2}$-terminal domain consisting of a hydrophobic signal sequence, a highly $O$-glycosylated tandem-repeat domain, a transmembrane domain, and a cytoplasmic domain $(4,9,10)$. Previous studies on MUC1 expression in colorectal carcinoma have chiefly focused on the tandem-repeat domain, and suggested that tumor cells expressing high levels of MUC1 may have increased invasive and metastatic potential (6). On the other hand, we focused on oligosaccharide moieties of MUC1 by using KL-6 antibody that requires sialylated oligosaccharides in addition to the polypeptide backbone as a part of antigen (11) and found that malignancy of colorectal adenocarcinoma such as invasion, metastasis, and worse prognosis of the patients, are correlated with a high level of KL-6 mucin expression in cytoplasm and circumferential cell membrane but not with the accumulation of KL-6 mucin on the apical side of the tumor cells (12).

Several colorectal carcinoma cell lines have been established with various morphology in culture and metastatic potential. COLO 201 cell line is characterized by formation of free cells in culture and by high metastatic potential (13). LoVo cell line is also characterized by high metastatic potential but forms adhesive cells in culture (14). In contrast, DLD-1 and WiDr cell lines are low metastatic and adhesive in culture $(15,16)$. Although KL-6 mucin was detected not only in various tumor tissues but also in tumor cell lines (17), expression and subcellular localization of KL6 mucin in these four cell lines has remained to be elucidated. In this study, we examined expression of KL-6 mucin in these colorectal carcinoma cell lines and describe that 
expression level and subcellular localization of KL-6 mucin may be related to the cell morphology in culture and metastatic potential in colorectal carcinoma cell lines.

\section{Materials and methods}

Cells and culture conditions. Human colorectal carcinoma cell lines (COLO 201, LoVo, WiDr, and DLD-1) were obtained from Japanese Collection of Research Bioresources, Osaka, Japan. COLO 201 and DLD-1 cells were cultured in RPMI1640 medium supplemented with $10 \%$ fetal bovine serum (FBS; Gibco, Grand Island, NY, USA). LoVo cells were cultured in Ham's F12 medium (Gibco) supplemented with $10 \%$ FBS. WiDr cells were cultured in Eagle's minimal essential medium containing non-essential amino acids (Gibco) and 10\% FBS. All cell cultures were carried out in a humidified atmosphere with $5 \% \mathrm{CO}_{2}$ in air at $37^{\circ} \mathrm{C}$. For maintenance and subculture, cells in exponential phase were treated with a mixture of $0.25 \%$ trypsin and $0.02 \%$ EDTA. To remove cell surface mucus, cells were incubated with $3 \%$ xylitol for $30 \mathrm{~min}$ at $22^{\circ} \mathrm{C}$ and then washed with PBS (18).

Western blot analysis. Western blotting was performed as described before (19). Briefly, cells pretreated with or without $3 \%$ xylitol were lysed in lysis buffer (50 mM Tris-HCl, $\mathrm{pH} 7.4$, containing $1 \%$ NP-40, $0.25 \% \mathrm{Na}$-deoxycholate, $150 \mathrm{mM}$ $\mathrm{NaCl}, 1 \mathrm{mM}$ EDTA, $1 \mathrm{mM}$ PMSF, $1 \mathrm{mM} \mathrm{Na} \mathrm{VO}_{4}, 1 \mathrm{mM}$ $\mathrm{NaF}$, and $1 \mu \mathrm{g} / \mathrm{ml}$ each of aprotinin, leupeptin, and pepstatin), sonicated and centrifuged at $15,000 \mathrm{~g}$ for $10 \mathrm{~min}$ at $4^{\circ} \mathrm{C}$. The cell lysates were resolved by SDS-PAGE $(8 \%$ gel) and transferred onto polyvinylidene difluoride membranes. After blocking the membranes in 5\% skim milk, the blots were probed with KL-6 antibody. After incubation with the secondary antibody conjugated with HRP, the reactivity was visualized by enhanced chemiluminescence using the ECL kit (GE Healthcare Bio-Sciences, Piscataway, NJ, USA).

Immunofluorescence and DNA staining. Cells $\left(1 \times 10^{6}\right)$ were fixed with $1 \%$ paraformaldehyde in PBS for $5 \mathrm{~min}$ at $4^{\circ} \mathrm{C}$ and, after two washes with PBS, incubated with or without KL-6 antibody (Eisai, Tokyo, Japan) in dilution of 1:200 for $60 \mathrm{~min}$. After two washes with PBS, cells were incubated with $2 \mu \mathrm{l}$ of FITC-conjugated secondary antibody (BD-Pharmingen, USA) for $20 \mathrm{~min}$ in the dark at room temperature. To monitor the cell condition, DNA staining was performed by incubating aliquots of the cell suspensions with PBS containing $50 \mu \mathrm{g} / \mathrm{ml}$ PI, $1 \mu \mathrm{g} / \mathrm{ml}$ RNase, $1 \%$ Triton $\mathrm{X}-100$ for $1 \mathrm{~h}$ at $4^{\circ} \mathrm{C}(20)$.

Flow cytometry. Flow cytometric examination was performed as described previously (20). Briefly, samples (10,000 cells) were analyzed by FACSCalibur flow cytometer (Becton Dickinson Immunocytometry Systems, San Jose, CA), with the CellQuest software for list mode acquisition and gated data analysis. In the bivariate analysis, $488 \mathrm{~nm}$ argon laser excitation $(15 \mathrm{~mW})$ was used for measurement of FITC fluorescence (FL1) with a 530/30 nm band-pass filter and PI fluorescence (FL2) with a $650 \mathrm{~nm}$ long-pass filter. FITC and PI fluorescence was measured using a logarithmic scale. Doublet discrimination mode was used for excluding the aggregation and debris of the cells.

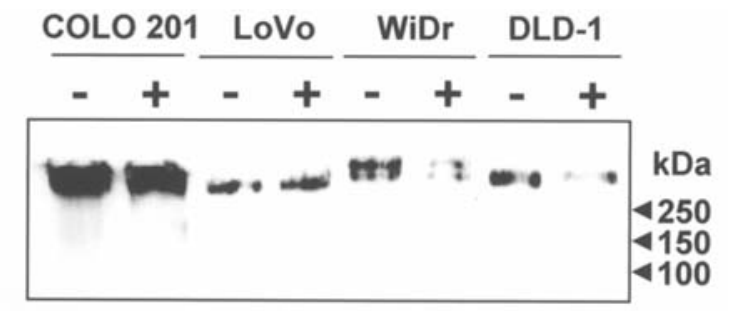

Figure 1. Western blot analysis of KL-6 mucin in colorectal carcinoma cell lines. COLO 201, LoVo, WiDr, and DLD-1 cells were treated with (+) or without (-) xylitol and then subjected to Western blot analysis as described in Materials and methods.

Immunocytochemistry. Cells cultured on slides were treated with or without $3 \%$ xylitol and fixed with $3.7 \%$ formaldehyde for 30 min followed by treatment with $0.2 \%$ Triton X-100 for $10 \mathrm{~min}$. Endogenous peroxidase activity was halted through administration of $0.3 \%$ hydrogen peroxide/methanol for $30 \mathrm{~min}$. The slides were rinsed with PBS, blocked with normal goat serum for $30 \mathrm{~min}$ at room temperature, and then incubated with or without KL-6 antibody (1:200 dilution) for $60 \mathrm{~min}$ at room temperature. After the incubation with biotinylated secondary antibody for $60 \mathrm{~min}$, bound biotinylated antibody was tested by the biotin-streptavidin-peroxidase complex method using a commercial kit (Histofine SAB-PO kit; Nichirei, Tokyo, Japan). 3,3'-Diaminobenzidine was used as the chromogen, and hematoxylin was used as a counterstain.

\section{Results}

Expression of KL-6 mucins in colorectal carcinoma cell lines. We performed Western blot analysis to examine KL-6 mucin expression in colorectal carcinoma cell lines, COLO 201, LoVo, WiDr, and DLD-1. As shown in Fig. 1, KL-6 mucins were detected at various molecular weights and number of molecular species. Notably, intensity of the bands in WiDr and DLD-1 cells significantly decreased when the cells were pretreated with a brief wash with xylitol.

Cell surface expression of KL-6 mucin. Cell surface expression of KL-6 mucin was examined by flow cytometric analysis after cells were treated with or without $3 \%$ xylitol. Fig. 2 shows typical flow cytometric profiles. A major population of COLO 201 cells was found to express a high level of KL-6 mucin on the cell surface and/or cytoplasm. Xylitol treatment did not alter the flow cytometric profile for KL-6 mucin expression, suggesting that KL-6 mucin detected in COLO 201 cells may tightly bind to the cell surface or exist in cytoplasm. In contrast, expression levels of KL-6 mucin in LoVo, WiDr, and DLD-1 cells were considerably lower than that in COLO 201 cells. KL-6 mucin levels on WiDr and DLD-1 cells but not on LoVo cells were decreased by xylitol treatment. This suggests that KL-6 mucin expressed in WiDr and DLD- 1 cells may be weakly attached to the cell membrane after the secretion of the cells.

Immunocytochemistry for KL-6 mucin in colorectal carcinoma cell lines. To further examine subcellular expression of KL-6 mucin, the cells with or without xylitol wash were treated with $0.2 \%$ Triton X-100 and then subjected to immunocytochemical 


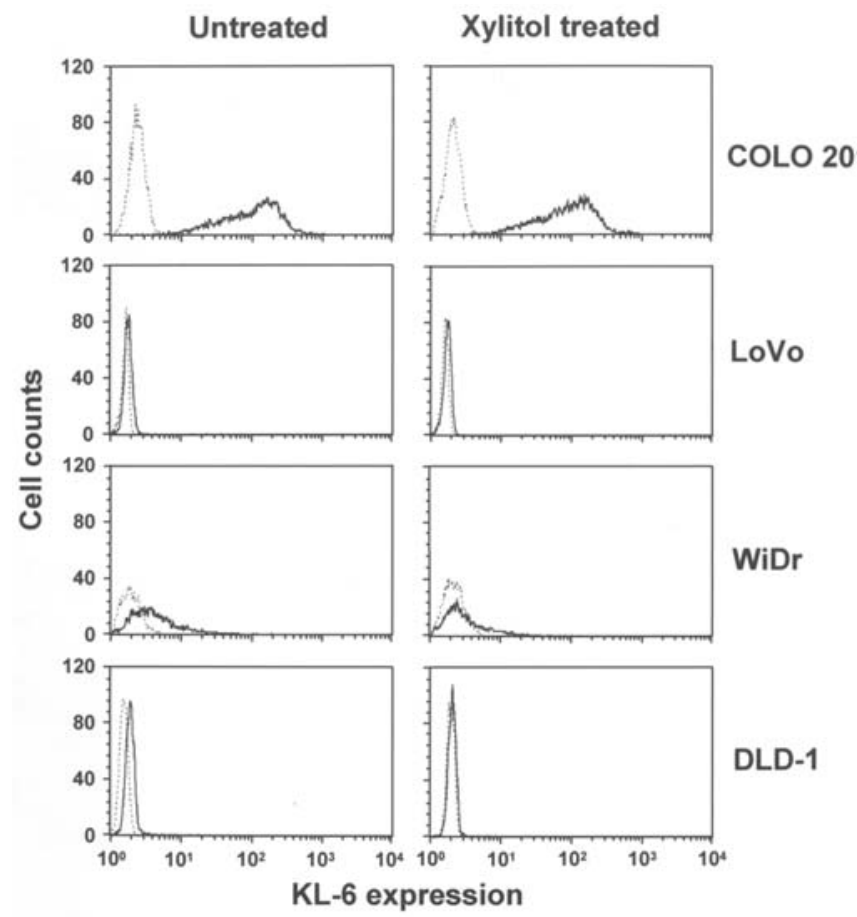

Figure 2. Flow cytometric analysis of cell surface KL-6 mucin in colorectal carcinoma cell lines. COLO 201, LoVo, WiDr, and DLD-1 cells were pretreated with (right panels) or without (left panels) xylitol and then subjected to flow cytometric analysis with (solid line) or without (dotted line) KL-6 antibody.

detection for KL-6 mucin (Fig. 3). In COLO 201 cells, even after xylitol treatment, intensive stains were observed in the entire cells including cell membrane and cytoplasm (Fig. 3A and B). In LoVo cells, weak stains were observed in the entire cells, especially in cytoplasm, which were not altered by xylitol treatment (Fig. 3C and D). In contrast, in WiDr and DLD-1 cells, moderate stains were observed around the cells without xylitol treatment (Fig. 3E and G, respectively). However, the stains in these two cell lines almost disappeared by xylitol treatment (Fig. 3F and $\mathrm{H}$ ).

\section{Discussion}

KL-6 mucin is a type of MUC1 mucin, which is detected by KL-6 monoclonal antibody $(6,11,17)$. In this study, we found that four colorectal carcinoma cell lines, COLO 201, LoVo, WiDr, and DLD-1, expressed KL-6 mucin and that the localization of KL-6 mucin was different among these cell lines. COLO 201 cells revealed extremely high level of KL-6 mucin in cytoplasm and cell surface where the mucin tightly bound without washing out by xylitol treatment. LoVo cells also revealed a similar localization of KL-6 mucin, although the expression level was considerably lower. WiDr and DLD-1 cells showed weak but significant expression of KL-6 mucin. In contrast to COLO 201 and LoVo cells, KL-6 mucins expressed by WiDr and DLD-1 cells were weakly attached onto the cell surface, since their mucin could be washed out by xylitol treatment.

Four colorectal carcinoma cell lines used in this study reveal different cell morphology in culture and metastatic potential (13-16). Cells in which a high level of KL-6 mucin

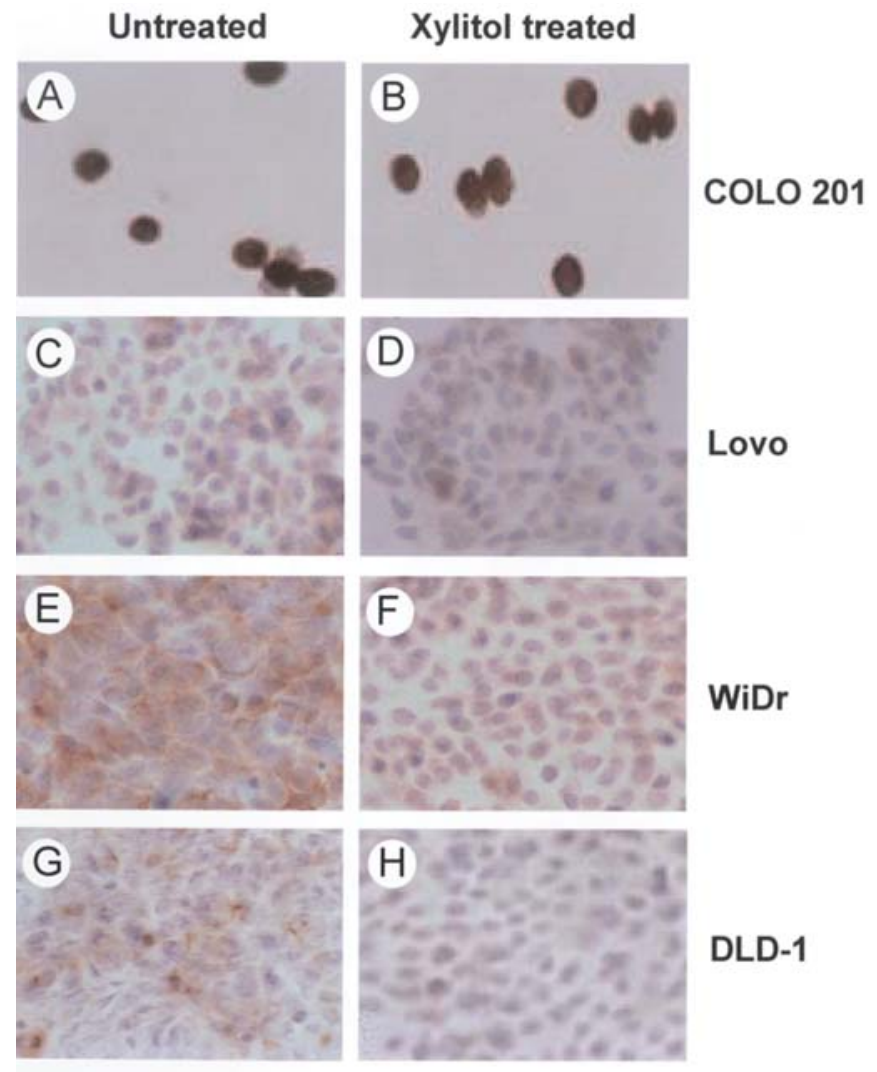

Figure 3. Immunocytochemical analysis of KL-6 mucin in colorectal carcinoma cell lines. COLO 201 (A and B), LoVo (C and D), WiDr (E and F), and DLD-1 (G and H) cells were pretreated with (right panels) or without (left panels) xylitol. Cells were then treated with $0.2 \%$ Triton X-100 and subjected to immunocytochemistry as described in Materials and methods. Original magnification: $\mathrm{x} 400$

tightly binds to cell surface may form free cells as revealed in COLO 201 cells. This may be due to physical effect of cell surface mucin to disturb cell-cell interaction (4). On the other hand, subcellular localization of KL-6 mucin may also be related to the malignancy of the cell lines. KL-6 mucin located in cytoplasm and cell surface of COLO 201 and LoVo cells, both of which are characterized by high metastatic potential, while KL-6 mucin weakly attached on cell surface of WiDr and DLD-1 cells, both of which are characterized by low metastatic potential, where the mucin could be removed by a treatment with xylitol. This suggests that KL-6 mucin may tightly bind to cell membrane and/or it exists in the cytoplasm of the high metastatic cell lines, while KL-6 mucin may weakly attach around the cell surface in the low metastatic cell lines.

We previously performed clinicopathological and immunohistochemical analyses for KL-6 mucin expression in colorectal adenocarcinoma tissues (12). The tumors could be categorized into three groups according to subcellular expression profiles of KL-6 mucin: 1) positive expression in circumferential membrane and/or cytoplasm, 2) positive expression only around apical surface, and 3) negative. Statistical analyses showed that circumferential and/or cytoplasmic expression of KL-6 mucin, but not its secretion around apical surface, was significantly correlated with lymph node and/or liver metastases, suggesting that subcellular localization of KL-6 mucin may be related to metastatic potential of the tumor (12). In the present study with colorectal carcinoma cell lines, we 
found that subcellular location of KL-6 may be related to the metastatic potential, which was consistent with our previous results on colorectal adenocarcinoma tissues. Studies on breast and lung carcinomas have also showed that subcellular localization of MUC1 rather than the level of its overall expression has a significant relationship with the metastasis $(21,22)$.

It has been discussed that cell surface MUC1 may play a role in the metastatic procedure, since the protruded structure of MUC1 may interfere with cell-cell adhesion and promote cell release from tumor body $(4,23)$ and since the cytoplasmic tail of MUC1, which is highly conserved among MUC1 species (24), may take part in the intracellular signaling such as $B$-catenin signaling pathway and promote tumor cell invasion (25). This means that the cell surface and cytoplasmic KL-6 mucin, a type of MUC1, may also play similar roles in the metastatic procedure. Identifying the underlying role that KL-6 mucin plays in the progressive behavior of the tumor cells needs further investigation.

In this study, xylitol was used to remove mucinous glycoproteins from cell surface $(18,26)$. The data obtained by Western blot analysis, flow cytometry, and immunocytochemistry suggested that the approach of xylitol wash was effective in removing KL-6 mucin from the cell surface. This would be a key step to elucidate the role of subcellular localization of MUC1 in the metastatic potential of the cell lines.

In conclusion, we suggest that the expression level and subcellular localization of KL-6 mucin may be related to the cell morphology in culture and metastic potential in colorectal carcinoma cell lines. In addition, xylitol is an effective tool to remove KL-6 mucin weakly attached around the cell surface, and to investigate the role of subcellular localization of KL-6 mucin.

\section{Acknowledgements}

This study was supported by Grants-in-Aid from the Ministry of Education, Science, Sports and Culture of Japan.

\section{References}

1. Gendler SJ and Spicer AP: Epithelial mucin genes. Ann Rev Physiol 57: 607-634, 1995.

2. Hey NA, Graham RA, Seif MW and Aplin JD: The polymorphic epithelial mucin MUC1 in human endometrium is regulated with maximal expression in the implantation phase. J Clin Endocrinol Metab 78: 337-342, 1994.

3. Hilkens J and Buijs F: Biosynthesis of MAM-6, an epithelial sialomucin. J Biol Chem 263: 4215-4222, 1998.

4. Hollingsworth MA and Swanson BJ: Mucins in cancer: protection and control of the cell surface. Nature Rev 4: 45-60, 2004.

5. Nakamori S, Ota DM, Cleary KR, Shirotani K and Irimura T: MUC1 mucin expression as a marker of progression and metastasis of human colorectal carcinoma. Gastroenterology 106: 353-361, 1994.

6. Hiraga Y, Tanaka S, Haruma K, Yoshihara M, Sumii K, Kajiyama G, Shimamoto F and Kohno N: Immunoreactive MUC1 expression at the deepest invasive portion correlates with prognosis of colorectal cancer. Oncology 55: 307-319, 1998.

7. Kashiwagi H, Kijima H, Dowaki S, Ohtani Y, Tobita K, Tsukui M, Tanaka Y, Matsubayasi H, Tsuchida T, Yamazaki H, Nakamura M, Ueyama Y, Tanaka M, Tajima T and Makuuchi H: DF3 expression in human gallbladder carcinoma: significance for lymphatic invasion. Int J Oncol 16: 455-459, 2000.
8. Luttges J, Feyerabend B, Buchelt T, Pacena M and Kloppel G: The mucin profile of noninvasive and invasive mucinous cystic neoplasms of the pancreas. Am J Surg Pathol 26: 466-471, 2002.

9. Gendler SJ, Lancaster CA, Taylor-Papadimitriou J, Duhig T, Peat N, Burchell J, Pemberton L, Lalani EN and Wilson D: Molecular cloning and expression of human tumor-associated polymorphic epithelial mucin. J Biol Chem 265: 15286-15293, 1990.

10. Lan MS, Batra SK, Qi WN, Metzgar RS and Hollingsworth MA: Cloning and sequencing of a human pancreatic tumor mucin cDNA. J Biol Chem 265: 15294-15299, 1990.

11. Kohno N, Inoue Y, Hamada H, Fujioka S, Fujino S, Yokoyama A, Hiwada K, Ueda N and Akiyama M: Difference in sero-diagnostic values among KL-6-associated mucins classified as cluster 9. Int J Cancer 8 (Suppl 1): 81-83, 1994.

12. Guo Q, Tang W, Inagaki Y, Midorikawa Y, Kokudo N, Sugawara Y, Nakata M, Konishi T, Nagawa H and Makuuchi M: Clinical significance of subcellular localization of KL-6 mucin in primary colorectal adenocarcinoma and metastatic tissues. World J Gastroenterol 12: 54-59, 2006.

13. Horiguchi N, Arimoto K, Mizutani A, Endo-Ichikawa Y, Nakada $\mathrm{H}$ and Taketani S: Galectin-1 induces cell adhesion to the extracellular matrix and apoptosis of non-adherent human colon cancer Colo201 cells. J Biochem (Tokyo) 134: 869-874, 2003.

14. Aznavoorian S, Liotta LA and Kupchik HZ: Characteristics of invasive and noninvasive human colorectal adenocarcinoma cells. J Natl Cancer Inst 82: 1485-1492, 1990.

15. Vermeulen SJ, Bruyneel EA, Bracke ME, De Bruyme GK, Vennekens KM, Vleminckx KL, Berx GJ, van Roy FM and Mareel MM: Transition from the noninvasive to the invasive phenotype and loss of alpha-catenin in human colon cancer cells. Cancer Res 55: 4722-4728, 1995.

16. Kuo TH, Kubota T, Watanabe M, Furukawa T, Teramoto T, Ishibiki K, Kitajima M, Moossa AR, Penman S and Hoffman R: Liver colonization competence governs colon cancer metastasis. Proc Natl Acad Sci USA 92: 12085-12089, 1995.

17. Kohno N: Monoclonal antibodies to human pulmonary carcinoma and their application to clinical diagnosis. Hiroshima J Med Sci 33: 971-996, 1985.

18. Sajjan U, Keshavjee S and Forstner J: Responses of welldifferentiated airway epithelial cell cultures from healthy donors and patients with cystic fibrosis to Burkholderia cenocepacia infection. Infect Immun 72: 4188-4199, 2004.

19. Guo Q, Tang W, Kokudo N, Sugawara Y, Miki K, Karako H, Qu X, Nakata M, Fujita-Yamaguchi Y and Makuuchi M: Epidermal growth factor-mediated growth control of confluent mammary epithelial cells cultured on artificial basement membrane. Int J Mol Med 16: 395-399, 2005.

20. Guo Q, Tang W, Mafune K, Yu J, Liao X, Li M, Wang X, Sugawara Y, Kokudo N and Makuuchi M: An in vitro evaluation of radiation effects of different fractionated regimens by absolute cell count beads. Oncol Rep 10: 1405-1410, 2003.

21. Rahn JJ, Dabbagh L, Pasdar M and Hugh JC: The importance of MUC1 cellular localization in patients with breast carcinoma: an immunohistologic study of 71 patients and review of the literature. Cancer 91: 1973-1982, 2001.

22. Guddo F, Giatromanolaki A, Koukourakis MI, Reina C, Vignola AM, Chlouverakis G, Hilkens J, Gatter KC, Harris AL and Bonsignore G: MUC1 (episialin) expression in non-small cell lung cancer is independent of EGFR and c-erbB-2 expression and correlates with poor survival in node positive patients. J Clin Pathol 51: 667-671,1998.

23. Kohlgraf KG, Gawron AJ, Higashi M, Meza JL, Burdick MD, Kitajima S, Kelly DL, Caffrey TC and Hollingsworth MA: Contribution of the MUC1 tandem repeat and cytoplasmic tail to invasive and metastatic properties of a pancreatic cancer cell line. Cancer Res 63: 5011-5020, 2003.

24. Ceriani RL, Chan CM, Baratta FS, Ozzello L, DeRosa CM and Habif DV: Levels of expression of breast epithelial mucin detected by monoclonal antibody BrE-3 in breast-cancer prognosis. Int J Cancer 51: 343-354, 1992.

25. Brayman M, Thathiah A and Carson DD: MUC1: a multifunctional cell surface component of reproductive tissue epithelia. Reprod Biol Endocrinol 2: 4, 2004.

26. Sajjan U, Moreira J, Liu M, Humar A, Chaparro C, Forstner J and Keshavjee S: A novel model to study bacterial adherence to the transplanted airway: inhibition of Burkholderia cepacia adherence to human airway by dextran and xylitol. J Heart Lung Transplant 23: 1382-1391, 2004. 